\title{
Bildung im Betrieb? Empirische Betrachtung der Bedarfsbestimmung im Großunternehmen: Prozesse, Akteure und Begründungen.
}

\author{
Tina Röbel (D)
}

Eingegangen: 12. Januar 2017 / Angenommen: 13. März 2017 / Online publiziert: 27. März 2017

(C) Der/die Autor(en) 2017. Dieser Artikel ist eine Open-Access-Publikation.

Zusammenfassung Dieser Beitrag widmet sich der betrieblichen Weiterbildung. Das Ziel ist dabei nicht, normative Forderungen an mögliche Bildungsprozesse zu formulieren, sondern vielmehr die Bedingungen, unter denen diese Prozesse stattfinden, empirisch zu betrachten. Der Fokus liegt auf dem Prozess der Bedarfsbestimmung, die einen Teil der Programmplanung darstellt. Es wird erstens beschrieben, wie dieser Prozess für verschiedene Programmarten abläuft und welche Akteure beteiligt sind. Zweitens wird in Anlehnung an die Kontexturanalyse explorativ untersucht, wie Bedarfsentscheidungen begründet werden. Die betrachteten Großunternehmen werden dabei nicht als homogene Organisationen beschrieben, vielmehr zeigen sich Unterschiede innerhalb der einzelnen Unternehmen und zwischen ihnen.

Als Datengrundlage dienen neun Interviews aus drei verschiedenen Unternehmen.

Schlüsselwörter Betriebliche Weiterbildung · Programmplanung ·

Unternehmensfallstudien $\cdot$ Bedarf $\cdot$ Akteure $\cdot$ Kontexturanalyse

\section{Education at the workplace? Empirical analysis of needs assessment in large enterprises: processes, stakeholders and justifications.}

\begin{abstract}
This article is devoted to workplace learning. Rather than formulating normative claims regarding educational processes, the aim is to empirically examine the conditions under which workplace learning takes place. The focus is on the process of needs assessment as part of program planning. Firstly, this process and the stakeholders involved are described for different types of programs. Secondly, an explorative analysis based on the contextual analysis shows how demand decisions are justified. The companies considered in this article are not described as homoge-
\end{abstract}

T. Röbel, M.A. ( $\bowtie)$

Humboldt-Universität zu Berlin, Berlin, Deutschland

E-Mail: tina.roebel@hu-berlin.de 
neous organizations, but differences are shown within the individual companies and between each other. A total of nine interviews from three different companies were selected for the analysis.

Keywords Workplace learning - Program planning · Case studies · Needs · Stakeholders · Contextual analysis

\section{Einleitung}

Wo gibt's denn sowas, dass die Personalentwicklung sagt ja du darfst 'nen Coach haben ja oder nein. Ne. Das hat die Führungskraft zu bestimmen, ob der 'n Coach kriegt oder nicht. [I: mhm] Wir können ihn beraten. Coach A B C D E. Das kann man ja einfach machen. Aber ansonsten sind DIE verdienen das Geld letztlich und können dann auch sagen was sie damit machen wollen. [I: mhm] Wenn sie sagen sie wollen mit Affen im Zoo irgendein Führungskräftedings machen, ja bitteschön. Macht (Leitung WB Abteilung, Fall A).

Weiterbildung, also organisiertes Lernen nach dem Abschluss einer ersten Bildungsphase, findet $\mathrm{zu}$ großen Teilen im betrieblichen Kontext statt. Laut Adult Education Survey 2014 (AES) waren $70 \%$ (2012: 69\%) der erhobenen Weiterbildungsaktivitäten der betrieblichen Weiterbildung zuzuordnen (BMBF 2015, S. 21). ${ }^{1}$ Die betriebliche Weiterbildung lässt sich in den Reproduktionskontext Unternehmen (Schrader 2010, S. 276) einordnen. Dieser ist zum einen dadurch geprägt, dass das Unternehmen der zuständigen Abteilung den Auftrag erteilt, Weiterbildung anzubieten, und dafür Ressourcen bereitstellt. Zum anderen unterscheidet sich dieser Kontext von Volkshochschulen oder bspw. Umweltinitiativen dadurch, dass mit der Weiterbildung private Interessen verfolgt werden (ebd.). Weiterbildung ist somit nicht das primäre Ziel von Betrieben, sie gehört nicht zum Kerngeschäft, sondern findet als beigeordnete Bildung statt (Gieseke und Heuer 2011).

Ausgangspunkt dieses Beitrags ist die Frage, wie Betriebe der Aufgabe nachkommen, Bildung zu ermöglichen. Auf normativer Ebene gehört dazu bspw. die Forderung, Weiterbildung für verschiedene Mitarbeitende zugänglich zu machen, um individuelle Entfaltung, gesellschaftliche Teilhabe und Beschäftigungsfähigkeit zu ermöglichen (vgl. Herbrechter und Schrader 2016, S. 8). Tatsächlich zeigen Statistiken zur Teilnahme an Weiterbildung auf der Makroebene betrieblicher Weiterbildung (Käpplinger 2016a), dass Zugangschancen in der Praxis einer starken Selektion unterliegen. Es partizipiert eher, wer männlich statt weiblich ist (39 zu 31\%), keinen Migrationshintergrund (38\% zu 22\%) und einen hohen beruflichen Abschluss hat (mit Fach-/Hochschulabschluss 49\%, mit Lehre 32\%; Bilger et al. 2013).

\footnotetext{
1 Betriebliche Weiterbildung im Sinne des AES wird über die zwei Merkmale ,Weiterbildung während der Arbeitszeit“" und/oder „Weiterbildungskostenübernahme durch den Arbeitgeber“ definiert (BMBF 2015, S. 19). In den diesem Artikel zugrunde liegenden Daten wurden Weiterbildungsmaßnahmen berücksichtigt, „die vorausgeplantes, organisiertes Lernen darstellen“ (Destatis 2013).
} 
Anstelle normativer Forderungen scheint daher für diesen Artikel eine empirische Betrachtung vielversprechender. Um zu verstehen, wie Unternehmen ihrer Aufgabe nachkommen, Bildung zu ermöglichen, wird untersucht, unter welchen Bedingungen Weiterbildung im Betrieb stattfindet. Dabei liegt der Fokus auf dem Prozess der Bedarfsbestimmung als Teil der betrieblichen Programmplanung, also auf dem „Planungs-, Management- und Entscheidungsverhalten von verantwortlichen Personen in weiterbildenden Betrieben“" (Käpplinger 2016a, S. 10). Bedarfsbestimmung kann als Kern pädagogischen Handelns verstanden werden, hier werden Ziele, Zielgruppen und Themen festgelegt.

Das Erkenntnisinteresse dieses Beitrags ist zweierlei. Zum einen soll der Prozess der Bedarfsbestimmung empirisch beschrieben werden. Zum anderen soll hinterfragt werden, welche Art von Begründungen bei den getroffenen Entscheidungen eine Rolle spielen.

Dabei wird von den folgenden Vorannahmen ausgegangen: Erstens, Weiterbildungsbedarf existiert nicht für sich und muss lediglich erhoben werden - weshalb der viel verwendete Begriff der Bedarfserhebung irreführend ist. Weiterbildungsbedarf ist vielmehr das Ergebnis eines Aushandlungsprozesses (vgl. Arnold 1991; Faulstich 1998; Gieseke 2008).

Weiterbildungsbedarfe haben also etwas mit unterschiedlichen Perspektiven, Verwertungs- und Nutzungsinteressen zu tun. Die Objektivierbarkeit bleibt immer rückgebunden an komplexe Entscheidungsprozesse, welche die jeweils Verantwortlichen treffen (Gieseke 2008, S. 29 f.).

Zweitens, es wird davon ausgegangen, dass man nicht von der betrieblichen Weiterbildung sprechen kann. Es muss zwischen Unternehmen und auch innerhalb eines Unternehmens differenziert werden. So existieren bspw. innerhalb der Unternehmen verschiedene Programmarten, die sich hinsichtlich Zielgruppen und zeitlichen Planungshorizonten unterscheiden (von Hippel und Röbel 2016; von Hippel 2016). ${ }^{2}$

Drittens, es wird angenommen, dass Programmplanung in der betrieblichen Weiterbildung in einem Spannungsfeld stattfindet, das u. a. von pädagogischen Zielen und ökonomischen Interessen geprägt ist. Unternehmen als solche sind sehr unterschiedlichen Einflüssen ausgesetzt, wie bspw. die Wettbewerbssituation, Produktveränderungen, Arbeitsmarktlage etc.

\section{Forschungslage und theoretischer Rahmen}

Der Beitrag ist in der Programmforschung verortet. Darüber hinaus sind auch Arbeiten zu Bedarf und Bedarfserhebungen, sowie eigene theoretische Vorarbeiten zur Begründung von Weiterbildungsentscheidungen wichtig.

\footnotetext{
2 Von Hippel und Röbel (2016) definieren fünf Programmarten: Programmkatalog, bereichsinterne Angebote, maßgeschneiderte Angebote, externe Angebote und weitere Einzelangebote.
} 


\subsection{Programmplanung in der betrieblichen Weiterbildung}

Anders als in der Betriebswirtschaft wird in der Erwachsenenpädagogik Programmplanung als interaktives „Angleichungshandeln“ (Gieseke 2008) verstanden, an dem mehrere inner- und außerbetriebliche Akteure beteiligt sind (vgl. Käpplinger 2016b). Betriebswirtschaftliche Zugänge gehen eher von linearen Planungszyklen aus (vgl. Käpplinger 2016a, S. 11 f.).

Das Planungshandeln ist dadurch geprägt, dass Weiterbildung nicht das primäre Ziel von Betrieben ist, sondern als beigeordnete Bildung stattfindet (Gieseke und Heuer 2011). Das bedeutet, dass sie aus Sicht der Unternehmen kontingent ist und ihren Nutzen über die Bildungswirkung hinaus begründen muss (u. a. Heuer 2010; Weber 1985). Dabei kommen unterschiedliche Handlungslogiken zum Tragen (Herbrechter und Schrader 2016, S. 2).

Weiterbildungsentscheidungen werden von den Interessen der an der Planung beteiligten Akteure beeinflusst (Käpplinger 2013; von Hippel und Röbel 2016). Neben der Personalentwicklung entscheiden auch Führungskräfte verschiedener Ebenen, Betriebsräte, Mitarbeiterinnen und Mitarbeiter und andere Personalbereiche, z. B. HR Business Partner, mit (von Hippel und Röbel 2016). Dabei kommen immer wieder unterschiedliche Akteurskonstellationen zustande, die sich auch als Konfigurationen bezeichnen lassen (Käpplinger 2013; 2016a; 2016b). Weber verweist bereits 1985 darauf, dass das Ergebnis betrieblicher Entscheidungsprozesse sowohl durch Kontextfaktoren, wie der Anzahl der Mitarbeiter des Betriebs, als auch von politischen Aspekten beeinflusst wird, wie z. B. der subjektiven Einschätzung der Situation durch die beteiligten Akteure (ebd., S. 36, vgl. auch Weber et al. 1994; sowie Cervero und Wilson 1994 zum Einfluss von Macht und Interessen).

Darüber hinaus wird Weiterbildung im betrieblichen Kontext eine Vielzahl verschiedener Funktionen zugeschrieben. Neben der Qualifizierungsfunktion lassen sich sowohl weitere Funktionen mit hoher Relevanz der Lernergebnisse, wie z. B. die Bewältigungs- und Flexibilitätsfunktion, als auch Funktionen mit geringer Relevanz der Lernergebnisse, wie z. B. die Gratifikations-, Motivations- und Mitarbeiterbindungsfunktion identifizieren (vgl. ausführlich von Hippel und Röbel 2016). Die im Rahmen der Programmplanung getroffenen Entscheidungen können somit als hochgradig kontingent und heterogen beschrieben werden (Käpplinger 2016a, S. 10).

\subsection{Bedarfsbestimmung in der betrieblichen Weiterbildung}

Weiterbildungsmanager gestalten Weiterbildung vor dem Hintergrund ihrer Situationsinterpretation, persönlicher Interessen und der internen Machtkonstellationen. Sie konfigurieren Weiterbildung auf der Grundlage vorgefundener Rahmenbedingungen und richten ihr Handeln daran aus. Im Mittelpunkt des Konfigurationsinteresses steht die Definitionsmacht über die Auslegung von Bildungsbedarf (Robak 2012, S. 188, Hervorh. d. Verf.).

Den Bedarf zu erkunden und zu bestimmen (andere Autoren sprechen von Bedarfserhebung, -erschließung, -ermittlung, -entwicklung, usw.) gehört zu den zentralen pädagogischen Aufgaben im Rahmen der Programmplanung (vgl. Robak 2012, 
S. 188; Reich-Claassen und von Hippel 2009, S. 1004; Gieseke 2008, S. 28; Seusing und Bötel 2000, S. 23). Die Bedarfsbestimmung ist somit Grundlage und oft auch Ausgangspunkt für die Ermöglichung von Lehr- und Lernprozessen im Betrieb.

In Abgrenzung zum Begriff „Bedürfnis“, der als subjektive Größe verstanden wird, wird „Bedarf“ als vermeintlich objektive Größe betrachtet (Gieseke 2008, S. 29). Zudem wird Bedarf als eher zielgerichtet verstanden. Der Begriff unterstellt, dass ein Angebot denkbar ist, das das Bedürfnis befriedigen kann (Schlutz 2006, S. 41). Während Bedürfnisse definitionsgemäß nur auf der individuellen Ebene auftreten können, kann Bedarf sowohl individuell als auch institutionell verstanden werden (ebd., S. 45). Gleichzeitig ist Bedarf auch gegenüber der Nachfrage abzugrenzen. ${ }^{3}$

Während die einfachsten Definitionen Bedarf als „Diskrepanz zwischen Ist und Soll im Verhalten bzw. der Leistung einer Person im Unternehmen“ (Grüner 2000, S. 106) interpretieren, verweisen andere Autoren darauf, dass Bedarf fälschlicherweise als konkret verstanden wird. Bereits die Wahl der Instrumente der Bedarfserhebung beeinflusst die Ergebnisse (vgl. Arnold 1991, S. 146 ff.; Faulstich 1998, S. 105 ff.; Jechle und Kolb 1994, S. 9). Dabei sind nicht nur Qualifikationsdefizite relevant, sondern auch die Bedürfnisse der Mitarbeiterinnen und Mitarbeiter, sowie die jeweilige Unternehmenskultur (vgl. „Bedarfsdimensionen“, Arnold und Wiegerling 1983, S. 34 f.; Arnold 1991, S. 148 f.), sowie die Interessenstruktur der Akteure (vgl. „,deskriptive Situationsanalysen“, Faulstich 1998, S. 114).

Damit ist bereits nachvollziehbar, dass Angebote nicht unmittelbar aus Bedarfserhebungen ableitbar sind, da Bedarfe nicht bereits auf bestimmte Angebote verweisen. Es schalten sich verschiedene interpretative Schritte ein, die bisher in der pädagogischen Forschung noch nicht bearbeitet wurden (Gieseke 2008, S. 34).

Empirische Arbeiten, die sich mit Bedarfserhebung beschäftigten, sind meist vorrangig quantitativ ausgerichtet. Sie zeigen, dass v. a. reaktive Verfahren oder einfache Soll-Ist-Vergleiche angewendet werden und stärker strategische Bedarfsermittlung selten ist (Seusing und Bötel 2000, S. 29; Grüner 2000, S. 148 f., Staudt 1990, S. 42). Überraschend ist, dass insgesamt zwar $73 \%$ der im CVTS befragten Unternehmen Weiterbildung anbieten, aber nur bei $21 \%$ der Unternehmen Bedarfsermittlung Teil des allgemeinen Planungsprozesses ist. Demgegenüber führen $42 \%$ der Unternehmen nur unregelmäßig Bedarfsermittlungen durch und $37 \%$ gar keine. ${ }^{4}$

Es ist also davon auszugehen, dass Bedarfe auch außerhalb von strukturierten Bedarfsermittlungsprozessen definiert werden. Wie das geschieht, welche Akteure beteiligt sind und wie die getroffenen Entscheidungen begründet werden, wurde bislang nicht detailliert untersucht (vgl. für eine Übersicht des Forschungsstands zu Bedarfserhebung Zalenska 2009, S. 116).

\footnotetext{
3 Schlutz unterscheidet zwischen latentem Bedarf und manifestem Bedarf (der nachgefragt wird) (Schlutz 2006, S. 41; vgl. auch Arnold und Wiegerling 1983, S. 37). Dabei kann Nachfrage auch dadurch entstehen, dass Bedarf durch ein Angebot induziert wird (Arnold und Lermen 2004, S. 11).

${ }^{4}$ Für frühere Jahre vgl. Seusing und Bötel (2000).
} 
Ähnlich wie für die gesamte Programmplanung lässt sich so die Lücke zwischen theoretisch ausgearbeiteten Bedarfserhebungsmethoden und deren tatsächlicher Nutzung in der Praxis erklären. Es fehlt an empirischer Analyse ,,̈̈ber das ganz konkrete Planungshandeln der betrieblichen Weiterbildungsverantwortlichen“ (Käpplinger 2010, S. 13).

\subsection{Begründungen von betrieblichen Weiterbildungsentscheidungen}

Die zweite Fragestellung dieses Artikels zielt auf die Begründungen betrieblicher Weiterbildungsbedarfe. Dabei soll bewusst eine dichotome Gegenüberstellung von pädagogischen und ökonomischen Begründungen vermieden werden (vgl. Röbel 2016). Vielmehr soll in Anlehnung an die Kontexturanalyse das Zusammenspiel verschiedener Begründungen aufgezeigt werden.

Damit kann an Arbeiten zu Begründungsmustern (Pohlmann 2015; Wittwer 1981), dem Einfluss von Macht und Interessen (Sork 1990; Cervero und Wilson 1994) und Modi der Aneignung betrieblicher Weiterbildung (Dörner 2008 über „Weiterbildungsbedeutungen“ als Bedingungen pädagogischen Handelns in kleinen und mittleren Unternehmen) angeschlossen werden. In Abgrenzung zu den bereits in 2.1 erwähnten zugeschriebenen Funktionen, bei denen es sich um unterstellte Zusammenhänge zwischen betrieblicher Weiterbildung und anderen Zielen handelt (von Hippel und Röbel 2016), können Begründungsmuster auch über das Unternehmen hinaus auf „Handlungs- und Wertmaßstäbe“ (Wittwer 1981, S. 231), wie „pädagogische Prinzipien“, und „gesellschaftspolitische Ansprüche“ (Pohlmann 2015, S. 100) verweisen.

\subsection{Fragestellungen und Ziele des Beitrags}

Ausgangspunkt des Beitrags ist die Frage, wie Betriebe als Organisationen ihrer Aufgabe nachkommen, Bildung zu ermöglichen. Dafür soll eine detaillierte empirische Betrachtung der Bedarfsbestimmung stattfinden. Diese zielt in einem ersten Schritt darauf ab, für verschiedene Programmarten den Prozessablauf und das $\mathrm{Zu}$ sammenspiel der Akteure beschreiben zu können. In einem zweiten Schritt wird in Anlehnung an die Kontexturanalyse explorativ betrachtet, welche Arten der Begründung für die getroffenen Entscheidungen herangezogen werden.

\section{Untersuchungsdesign}

\subsection{Sample und Datenerhebung}

Grundlage für die Untersuchung sind Daten aus einem DFG-Projekt zu Programmplanung in der betrieblichen Weiterbildung, das von 2013 bis 2015 an der HumboldtUniversität zu Berlin durchgeführt wurde (von Hippel und Röbel 2016). ${ }^{5}$ In allen drei untersuchten Großunternehmen (über 2800 Mitarbeiter, vgl. Bäumer 1999) existiert

\footnotetext{
5 Das Projekt wurde von der DFG unter dem Kennzeichen HI 1599/1-1 gefördert.
} 
eine Abteilung mit mehreren hauptamtlichen Stellen, die für Weiterbildung zuständig ist. Die Studie war branchenübergreifend (Gesundheitsbranche, Einzelhandel und Energie). Insgesamt wurden 35 Interviews mit verschiedenen Akteuren geführt. Für die Sekundäranalyse wurden je Fall drei, also insgesamt neun Interviews ausgewählt:

\section{Leitung der Weiterbildungsabteilung (Experteninterview)}

2. Mitarbeitende der Weiterbildungsabteilung (Fall A und C) bzw. regionale Personalentwickler (Fall B) (Experteninterview)

3. Führungskräfte einzelner Standorte/Bereiche (problemzentriertes Interview)

Die Sekundäranalyse hat mehrere Vorteile. Neben dem pragmatischen Vorteil, bereits Daten aus dem für erwachsenenpädagogische Forschung nur schwer zugänglichen Bereich der Großunternehmen vorliegen zu haben, ist auch mit weniger sozial erwünschten Antworten zu rechnen. Bedarf, Bedarfsbestimmung und -begründung standen nicht explizit im Vordergrund der Interviews. Anschlussforschung könnte in Form von teilnehmender Beobachtung an Planungsgesprächen stattfinden.

\subsection{Datenauswertung Fragestellung 1 (Inhaltsanalyse)}

Für die erste Fragestellung, die detaillierte Beschreibung der Bedarfsbestimmung, wurde inhaltsanalytisch (Mayring 2003) ausgewertet. Dafür wurden zunächst anhand der vollständigen Transkripte in MAXQDA den beiden Unterfragestellungen Textstellen zugeordnet:

1. Prozess der Bedarfsbestimmung (Was, wann, wie?)

2. Akteure der Bedarfsbestimmung (Wer ist wie beteiligt?)

Für beide Themen wurden im nächsten Schritt die zugeordneten Textstellen je Interview paraphrasierend zusammengefasst und eine thematische Übersicht erstellt. Anschließend wurde je Fall eine fallinterne vergleichende Zusammenfassung erstellt. Im letzten Schritt wurde ein fallübergreifender Vergleich durchgeführt.

\subsection{Datenauswertung Fragestellung 2 (Kontexturanalyse)}

Die zweite Fragestellung zielt auf die Begründungen der getroffenen Entscheidungen. Hier wurden explorativ zentrale Textstellen aus den Interviews mit den Weiterbildungsleitungen in Anlehnung an die Kontexturanalyse ausgewertet.

Die Kontexturanalyse wurde für die dokumentarische Organisationsforschung in Anschluss an die dokumentarische Methode entwickelt (vgl. Jansen et al. 2015). Kerngedanke ist, dass in Organisationen verschiedene Kontexturen aufeinandertreffen. Dazu gehören sowohl verschiedene Logiken (,multiple Rationalitäten“), sowie querliegend dazu verschiedene gesellschaftliche Funktionssyteme (ebd.). ${ }^{6}$ Eine Gegenüberstellung von lediglich zwei Kontexturen, z. B. Pädagogik und Ökonomie,

\footnotetext{
6 Vgl. auch Jansen et al. (2015): „Eine Kontextur kann dabei nahezu alles sein: eine Person, eine Organisation, eine Reflexionsinstanz. Was als eine solche funktioniert, bleibt damit dem empirischen Material überlassen“" (ebd., S. 23).
} 
würde aus dieser Perspektive zu kurz greifen. Vielmehr finden alle Kontexturen gleichzeitig statt („Kopräsenz unterschiedlicher logischer Räume“). Interessant ist, wie die einzelnen Kontexturen aufeinander bezogen werden, also „wie im je konkreten Fall ein Arrangement verschiedener Kontexturen geschaffen wird, das eine Kollision vermeidet und Entscheidungsfindung sicherstellt“ (ebd.). Beispiele für Datenauswertungen mit Hilfe der Kontexturanalyse finden sich bei Vogd (2009), Vogd et al. (2015) und Lüdecke (2016).

Das Verfahren orientiert sich an der komparativen Analyse der dokumentarischen Methode. Nach einer formulierenden Interpretation, die thematische Verläufe skizziert und Textpassagen inhaltlich zusammenfasst, erfolgt eine reflektierende Interpretation. Dabei werden angesprochene Problemstellungen, sowie dazugehörige Orientierungsrahmen herausgearbeitet (vgl. Lüdecke 2016). Im Anschluss wird während der komparativen Analyse analysiert, bei welchen Problemstellungen und in welchen Situationen auf welche Kontexturen (nicht) Bezug genommen wird. Es werden ,die verschiedenen Logiken in ihrer wechselseitigen Relationierung“ beobachtet (Jansen et al. 2015).

Beispielsweise mag ein ärztlicher Entscheidungsprozess darin einrasten, einen Patienten aus Kostengründen vorschnell zu entlassen. Dies heißt jedoch nicht, dass die nicht thematisierten Kontexturen hiermit aus dem Spiel sind ... Es wäre also nach Pannen und Störungen zu suchen, welche die impliziten Erwartungshorizonte deutlich werden lassen, deren Befolgung auch dann erwartet wird, wenn in der Kommunikation überwiegend andere Themen verhandelt werden (Vogd 2009).

Da die Interviews leitfadengestützt durchgeführt wurden, sind vor allem längere Passagen, in denen die Interviewpartner ,,ihre eigene Relevanzstruktur“ entfalten können (Vogd et al. 2015), interessant. Gleichzeitig gilt es, die Interviewsituation mit zu reflektieren und zu prüfen, welche Kontexturen durch die Interviewleitung eingeführt, betont oder möglicherweise auch ignoriert wurden (zum Einfluss des Interviewers vgl. John 2010).

\section{Ergebnisse}

\subsection{Prozesse und Akteure der Bedarfsbestimmung}

Die Bedarfsbestimmung unterscheidet sich in allen drei Unternehmen je nach Programmart und wird deshalb jeweils gesondert beschrieben.

Programmkatalog. In Bezug auf die von der Weiterbildungsabteilung (WBA) im Voraus geplanten, offenen Angebote werden in allen Unternehmen zwei Prozesse der Bedarfsbestimmung unterschieden. Zum einen gibt es quantitative Planungen, bei denen die Nutzung der Angebote im Vorjahr als Bedarf angenommen wird. Dabei werden die unterschiedlichen Motive der Nutzung kritisch reflektiert, wie z. B. ,Trainings als Incentive, weil der Mitarbeiter fleißig war" gegenüber , wirklich wertstiftend" (Leitung WBA Fall C). Zum anderen gibt es Wege und Verfahren, 
wie neue Angebote entstehen. Diese sind bei Fall A und B größtenteils informell, zufällig und von persönlichen Kontakten abhängig. Die Gründe dafür sind unterschiedlich. Bei Fall A reicht das Geld nicht für ,,dieses wunschinduzierende, na was hättet ihr denn gerne" (Leitung WBA Fall C). Bei Fall B verfolgt man das Ziel, das veranstaltete Lernen „nach und nach abzuschaffen“ (Leitung WBA Fall B). Demgegenüber finden bei Fall $\mathrm{C}$ jährliche Planungsgespräche mit Vertretern der Tochterunternehmen statt, in denen neue Themen abgefragt werden. Darüber hinaus setzt der Gesetzgeber durch Vorschriften Bedarfe, z. B. bzgl. Ersthelfer, Brandschutz oder Sicherheitsaspekten. Diese Art von Bedarfen wird in der Regel von den Fachabteilungen erkannt.

Ich kann ja nicht alle Bestimmungen in dieser Welt wissen (Leitung WBA Fall A).

Maßgeschneiderte Angebote. Hierunter fallen Angebote, bei denen die WBA auf kurzfristige Bedarfe aus unterschiedlichen Unternehmensbereichen reagiert. In der Rolle eines Dienstleisters führt sie teilweise Angebote selbst durch oder vermittelt zwischen den internen Kunden und externen Anbietern. In Unternehmen A und C wird von dieser Programmart deutlich mehr berichtet als bei Fall B. Es gibt jeweils zwei verschiedene Abläufe. Teilweise werden Bedarfe im Vorhinein besprochen und geplant, z. B. in viertel-/jährlichen Planungsgesprächen mit einzelnen Unternehmensbereichen, an denen verschiedene Führungskräfte beteiligt sind. Häufiger scheint es vorzukommen, dass kurzfristig eine Führungskraft die WBA kontaktiert, um Unterstützung anzufragen. In den WBA bei Fall A und C weiß man, dass der Prozess der Bedarfsbestimmung ,schon sehr auch von so' nem Zufallssytem abhängig [ist]. Die is' sehr von Beziehungen abhängig, die is' wenig institutionalisiert“ (Mitarbeiter WBA Fall C).

Interessant ist die Frage, wer die Kompetenz hat den Bedarf ,richtig“ zu erfassen. Die Mitarbeiter der WBA von Fall A und C betonen die Notwendigkeit von Auftragsklärungsgesprächen, weil der Kunde nicht immer das braucht, was er sagt. Dem widersprechend berichtet eine Führungskraft, dass sie die Abstimmung mit dem externen Anbieter selbst übernommen hat, weil die Vorschläge der WBA den Bedarf nicht getroffen haben.

Sie sind so überrascht dass ich das tue? (.) Wer soll es denn sonst tun? Ich bin doch hier die Verantwortliche für den Standort. (.) Also. Da ist es ja ganz schnell passiert, dass die Maßnahmen, die sonst von von der Fort- und Weiterbildung kämen an dem Ziel völlig vorbeigehen (Führungskraft Fall A).

Bereichsinterne Angebote. In allen drei Unternehmen gibt es in verschiedenen Unternehmensbereichen bzw. Standorten eigenständig geplante Angebote ohne Beteiligung der WBA. In Fall A wird ein Teil des zentralen Budgets für Weiterbildung direkt den Standorten zugeteilt (Verteilungsschlüssel ist die Mitarbeitergröße) und kann für diese Angebote genutzt werden - nach Rücksprache mit der WBA. In Fall B spricht die Leitung WBA nicht über bereichsinterne Angebote. Aus Sicht der regionalen Personalentwickler, die solche Angebote auf Wunsch der Mitarbei- 
ter eingeführt haben, besteht kein Unterstützungsbedarf durch die WBA. In Fall C sieht die Leitung WBA die bereichsinternen Angebote kritisch, weil sie verhindern, dass Kosten gespart werden. Für die Führungskraft, die davon berichtet, scheinen sie selbstverständlich, es entsteht fast der Eindruck, dass die WBA überflüssig oder zumindest unbekannt ist.

Naja, so, wie, wie, wie läuft das ab? Also es gibt einmal 'n Aufgabenspektrum eines Bereiches, ne? [I: mhm] So dann gibt's die Leute, die da arbeiten, ja? ... So und dann stimmen wir uns hier intern, also ich mit meinen drei Abteilungsleitern, stimmen wir uns ab und sagen „Pass ma' auf, ja, wollen wir nich' ma' dort oder es wäre notwendig, dass wir dort mal die Leute ertüchtigen“" (Führungskraft Fall C).

Externe Angebote. Hierunter fallen sowohl Angebote, die von der WBA organisiert werden, indem z. B. Rahmenverträge mit Anbietern von Sprachkursen abgeschlossen werden, als auch externe Angebote, an denen einzelne Mitarbeitende teilnehmen - in diesem Fall entscheidet die jeweilige Führungskraft. Es scheint in keinem der Unternehmen strukturierte Prozesse zu geben, um Bedarf zu bestimmen.

In Fall A wird die eigenständige Nutzung externer Angebote aus zwei Gründen positiv gesehen. Erstens braucht man sich nicht darum zu kümmern, fachliche Bedarfe zu erkennen. Zweitens ist es eine Möglichkeit, mit dem als gering empfundenen Budget umzugehen. Die Verantwortung, Bedarfe zu erkennen bzw. Entscheidungen zu treffen, wird an die Bereiche übertragen.

... und deshalb hab ich's eben so gemacht, dass ich Teile dieses Budgets ... denen gebe und sag': ihr dürft daraus coachen, Teamentwicklung, die Leute zu Kongressen schicken, macht was ihr wollt - müsst ihr nur über uns machen, damit ich das controllen kann, weil ich das so sozusagen verantworte, aber das ist euer Ding und ihr entscheidet selber (Leitung WBA Fall A).

Weitere Einzelangebote. Hierzu gehören weitere Weiterbildungsangebote bzw. Aktivitäten der WBA, wie z. B. eine Kampagne zum Schutz vor sexualisierter Gewalt (Fall A). Die Bedarfsbestimmung erfolgt nicht strukturiert, die Initiative kann sowohl von der WBA als auch von anderen Akteuren ausgehen. Diese Einzelangebote können auch dazu dienen, neue Lernformate auszuprobieren, wie in Fall C, wo eine Summerschool angeboten wurde. Als Besonderheit sind die Führungskräftetrainings bei Fall C zu nennen, die zwar durch die WBA im Voraus geplante Angebote darstellen, aber nicht Teil des Programmkatalogs sind. Sie werden top-down von der Stabsstelle „Talent Management“ in der internationalen Konzernzentrale anhand der Führungswerte entwickelt. Aufgabe der WBA ist es, die Führungskräftetrainings ,möglichst reibungsfrei“ und kostensparend ,abzufahren“ (Mitarbeiter WBA Fall C). 


\subsection{Begründungen für Bedarfsentscheidungen}

Die zweite Fragestellung des Beitrags zielt auf die Begründungen betrieblicher Bedarfsentscheidungen. Dafür wurden explorativ Textpassagen aus den Interviews mit der Leitung WBA je Unternehmen in Anlehnung an die Kontexturanalyse ausgewertet. Im Mittelpunkt steht dabei jeweils ein einzelnes Weiterbildungsangebot: Yoga (Fall A, Programmkatalog), Fotografie (Fall B, Programmkatalog), Job-Flow-Trainer (Fall C, weiteres Einzelangebot). In allen drei Passagen folgt die Begründung des Angebots auf allgemeinere Überlegungen zu Weiterbildung. Für Fall A wird die Analyse ausführlicher dargestellt, anschließend folgt eine vergleichende Diskussion.

Sie müssen natürlich gucken, dass die Leute das Wissen haben, was sie brauchen um ihren Job zu machen. [I: ja] Ja, Sie müssen gucken, dass die Leute motiviert bleiben bei der Arbeit, dass sie sich entwickeln können, ... verstehen Sie das hat, ... was wir da bieten müssen, ist ja sehr abhängig wo 'ne Organisation gerade steht, [I: mh] wo sie hin will, strategisch, ja? Natürlich sind hier auch n'paar Goodies bei, „Hatha Yoga“, aber das ist auch entspannend. Und ich sag auch immer, wenn ich in Berlin bin, wissen Sie, ich gehe nicht oft ins Theater, ich hab ein Theaterabo. Aber die Tatsache, dass ich ins Theater gehen kann, [I: mhm] das gibt mir ein gutes Gefühl. Wie oft gehen die Leute ins Theater? Oder ins Kino? [I: okay, ich verstehe Sie, ja] Ja? Aber das, dass ich es könnte wenn ich es möchte, das gibt das Gefühl der Erfüllung, ja? (Leitung WBA Fall A).

Interessant an diesem Zitat ist der Widerspruch zwischen der abstrakten Beschreibung der strategischen Ausrichtung der Weiterbildungsarbeit und dem herausgegriffenen Angebot „Hatha Yoga“. Während es zunächst um Legitimation und Professionalität der eigenen Arbeit, sowie den Rückbezug zum Unternehmen geht, spricht sie mit dem Beispiel und der gewählten Metapher des Theaterbesuchs ganz andere Kontexturen an. Zunächst wird noch auf Effektivität und Gesundheit verwiesen (,,aber das ist auch entspannend“), also Kontexturen für die die Teilnahme am Weiterbildungsangebot bedeutsam ist. Im weiteren Verlauf der Passage scheint lediglich das Anbieten des Angebots relevant zu sein. Es geht um die Möglichkeit der Teilnahme, den vermeintlich leichten Zugang, ganz nach Bedürfnis. All das zielt auf Zufriedenheit (,Gefühl der Erfüllung “). Durch die vehemente Nachfrage „Wie oft gehen die Leute ins Theater? Oder ins Kino?" entsteht der Eindruck, dass die (Nicht-)Teilnahme von der Entscheidung der Mitarbeitenden abhängt und weniger wie es der Fall ist - von der Anzahl der Plätze oder der Zustimmung der jeweiligen Führungskraft.

Auch bei den anderen beiden untersuchten Passagen sprechen die Interviewpartner zunächst auf einer abstrakten Ebene. Es geht darum auf Veränderungen in der Umwelt (ständig neue Produkte im Handeln) bzw. auf hierarchisch kommunizierte Anforderungen (bzgl. Weiterbildungsmethoden) und Veränderungen innerhalb der Organisation (Umstrukturierung) zu reagieren. Ein Gestaltungsanspruch wird erst auf der Ebene der konkreten Angebote sichtbar.

(Fall B) Das gewählte Beispiel „Fotografie“ unterstreicht die Bedeutung der Weiterbildungsarbeit, man könnte auch sagen, es fordert Anerkennung ein. Die „Of- 
fensive" scheint wie ein heldenhafter Schutz der Mitarbeitenden in der Flut neuer Produkte (,die Berührungsängste waren riesig groß“). Weitere aufgefundene Kontexturen sind die Ehrfurcht vor Technik, das Betonen der eigenen Professionalität und des Erfolgs der Offensive (die Mitarbeiter können heute mit den Fotoautomaten umgehen). Darüber hinaus wird auch auf die Perspektive des Kunden eingegangen. Direkte Verweise zu wirtschaftlichen Überlegungen finden sich in dieser Passage nicht.

(Fall C) Die Interviewpartnerin betont gleich einleitend die guten Erfahrungen, die man mit dem Angebot „Job-Flow-Trainer“ gemacht hat, unterstreicht also die Professionalität ihrer Abteilung. In der Begründung der Vorteile des Angebots bezieht sie sich vor allem auf die Effektivität und Effizienz der Maßnahme für das Ziel der Qualifizierung und die dadurch besseren Ergebnisse (mehr Qualität und bessere Performance). Darüber hinaus verweist sie auf die Kontextur der Anerkennung der Mitarbeitenden ohne den Eindruck zu erwecken, dass das für sie sehr bedeutsam wäre „das hat auch was so mit Anerkennung und wie auch immer zu tun “.

\section{Diskussion und Ausblick}

Ausgangspunkt für diesen Beitrag war die Frage, wie Unternehmen als Organisationen ihrer Aufgabe nachkommen, Weiterbildung zu ermöglichen. Betriebliche Weiterbildung wurde dafür als beigeordnete Bildung definiert (Heuer und Gieseke 2011). Das Weiterbildungsprogramm wird von verschiedenen Akteure über Angleichungshandeln (Gieseke 2008) erstellt. Der Fokus des Beitrags lag auf dem Prozess der Bedarfsbestimmung. Dieser wurde in einem ersten Schritt empirisch detailliert für verschiedene Programmarten beschrieben. In einem zweiten Schritt wurden die Begründungen der Bedarfsentscheidungen explorativ analysiert.

Für die erste Fragestellung lässt sich zusammenfassend sagen, dass in allen drei Fällen für die Angebote des Programmkatalogs, sowie in Teilen bei den weiteren Einzelangeboten, die WBA die Haupt-Entscheidungsinstanz ist. Bei allen anderen Angeboten spielen Führungskräfte auf verschiedenen Hierarchieebenen eine wichtige Rolle. Sie sind Impulsgeber, Entscheider und teilweise auch Planer für maßgeschneiderte, bereichsinterne und externe Angebote, da sie die Budgetverantwortung tragen. Darüber hinaus entscheiden sie über die individuelle Teilnahme an (internen und externen) Angeboten. Eine direkte Einflussnahme der Geschäftsleitung gibt es lediglich bei Fall C auf die Führungskräftetrainings. Diese Ergebnisse decken sich mit der quantitativen Erhebung von Seusing und Bötel (2000). Insgesamt läuft Bedarfsbestimmung über alle Programmarten hinweg eher kommunikativ als instrumentell (vgl. Faulstich 1998, S. 114). Als weiterer analytischer Schritt wäre hier eine Typenbildung denkbar, um verschiedene Formen der Bedarfserschließung zu beschreiben. Mögliche Dimensionen sind die Beteiligung der WBA (ja/nein), die zeitliche Perspektive (kurz-/langfristig), die berücksichtigten Zielgruppen (exklusiv/ inklusiv), usw.

Im zweiten Schritt konnte anhand der explorativen Kontexturanalyse gezeigt werden, dass bereits einzelne Akteure, in diesem Fall die Leitungen WBA, auf sehr verschiedene Kontexturen Bezug nehmen. Dabei war vor allem interessant, dass 
sich Begründungen auf der Ebene betrieblicher Weiterbildung insgesamt von den Begründungen im Zusammenhang einzelner Angebote unterscheiden. Die denkbare Kontextur „Bildung“ oder spezifischer „Entwicklung individueller Anlagen“, ,gesellschaftliche Integration“ oder „Beschäftigungsfähigkeit“ (Herbrechter und Schrader 2016, S. 8) konnte in Bezug auf die drei betrachteten Angebote nicht gefunden werden, wenn auch die Leitung WBA in Fall A allgemein von der Notwendigkeit spricht, dass ,die Leute sich entwickeln können“. Versteht man die Frage nach Bildung als Verweis auf „Horizonte humaner Perspektive“ (Faulstisch und Zeuner 2015, S. 27), lassen sich dem durchaus Kontexturen zuweisen, auf die die Interviewpartner Bezug nehmen: Gesundheit, Mitarbeiterbedürfnisse, Schutz, Unterstützung, Anerkennung. Auf Grund des explorativen Charakters der Analyse können alle Aussagen nur Hypothesen sein, die durch weitere Arbeit am Material zu prüfen sind.

Einschränkend ist zu sehen, dass dieser Beitrag lediglich drei Interviewpartner je Unternehmen berücksichtigt hat. Dadurch blieben andere Akteure, wie bspw. der Betriebsrat, außen vor. Darüber hinaus wurde die Frage der Nutzung der Angebote (Zugang, Erkennen von individuellem Bedarf etc.) ausgeklammert. Dies sollte in weiteren Forschungsarbeiten berücksichtigt werden.

Für Anschlussforschung ist die wichtige Rolle der Führungskräfte bei der Planung von Weiterbildung interessant. Auch die verschiedenen Formen der Budgetallokation gehören in den Fokus erwachsenenpädagogischer Forschung, schließlich entscheidet sich daran, welche Akteure wieviel Einfluss auf Bedarfsentscheidungen nehmen (zu Planungspartizipation vgl. Robak et al. 2013). Zusätzlich wäre eine quantitative Erhebung vielversprechend, die die Bedeutung der verschiedenen Programmarten untereinander misst (Teilnahmezahlen, Dauer der Angebote, Budget etc.).

Methodisch ist die Kontexturanalyse als Auswertungsverfahren für weitere Forschung im Kontext betrieblicher Weiterbildung anschlussfähig. Hier ist insbesondere interessant zu prüfen, ob sich ein Zusammenhang zwischen den Funktionen, die einzelnen Programmarten zugeschrieben werden, und dem Prozess der Bedarfsbestimmung sowie den zur Begründung herangezogenen Kontexturen aufzeigen lässt. Dafür könnten Situationen, in denen ,,die formalen Seiten der Organisation unterlaufen werden“, besonders aufschlussreich sein (Harney 1996, S. 118). Darüber hinaus wäre auch eine teilnehmende Beobachtung an einem Planungsgespräch vielversprechend.

Mit Blick auf die Praxis betrieblicher Weiterbildung muss man Respekt haben vor denjenigen, die dort Weiterbildungsentscheidungen treffen. Ähnlich wie Ärzte sind sie mit sehr unterschiedlichen Akteuren und Kontexturen konfrontiert. Eine erwachsenenpädagogische Forschung, die dabei unterstützen möchte, Bildung zu ermöglichen, darf nicht nur normative Forderungen formulieren, sondern muss Verständnis zeigen für die Komplexität der Praxis. Es gilt, die verschiedenen Bedarfsbegründungen und ihr Zusammenspiel sichtbar zu machen.

Förderung Deutsche Forschungsgemeinschaft (DE): Kennzeichen HI 1599/1-1

Open Access Dieser Artikel wird unter der Creative Commons Namensnennung 4.0 International Lizenz (http://creativecommons.org/licenses/by/4.0/deed.de) veröffentlicht, welche die Nutzung, Vervielfältigung, Bearbeitung, Verbreitung und Wiedergabe in jeglichem Medium und Format erlaubt, sofern Sie den/die ursprünglichen Autor(en) und die Quelle ordnungsgemäß nennen, einen Link zur Creative Commons Lizenz beifügen und angeben, ob Änderungen vorgenommen wurden. 


\section{Literatur}

Arnold, R., \& Wiegerling, H.-J. (1983). Programmplanung in der Weiterbildung. Bedarfsorientierung ausgew. Planungsstrategien - institutionelle Einflïsse (1. Aufl.). Frankfurt am Main: Moritz Diesterweg.

Arnold, R. (Hrsg.). (1991). Taschenbuch der betrieblichen Bildungsarbeit. Hohengehren: Schneider.

Arnold, R., \& Lermen, M. (2004). Die Systematik des Bedarfs: „Es geht eigentlich um etwas ganz anderes“. Report. Zeitschrift für Weiterbildungsforschung, 27(2), 9-16.

Bäumer, J. (1999). Weiterbildungsmanagement. Eine empirische Analyse deutscher Unternehmen. Bd. 1. München: Hampp.

Bilger, F., Gnahs, D., Hartmann, J., \& Kuper, H. (2013). Weiterbildungsverhalten in Deutschland. Resultate des Adult Education Survey 2012. Bielefeld: Bertelsmann.

Bundesministerium für Bildung und Forschung (2015). Weiterbildungsverhalten in Deutschland. AES 2014 Trendbericht. https://www.bmbf.de/pub/Weiterbildungsverhalten_in_Deutschland_2014.pdf. Zugegriffen: 13. März 2017.

Cervero, R. M., \& Wilson, A.L. (1994). The politics of responsibility: a theory of program planning practice for adult education. Adult Education Quarterly, 45(1), 249-268.

Destatis (2013). Berufliche Weiterbildung in Unternehmen. Wiesbaden: Statistisches Bundesamt.

Dörner, O. (2008). Weiterbildungsbedeutungen: Ein Bedingungsbereich pädagogischen Handelns in kleinen und mittleren Unternehmen. Der pädagogische Blick, 3, 132-141.

Faulstich, P. (1998). Strategien der betrieblichen Weiterbildung. Kompetenz und Organisation. München: Vahlen.

Faulstich, P., \& Zeuner, C. (2015). Ökonomisierung und Politisierung des Feldes der Erwachsenenbildung: Die Rolle der Wissenschaft. Erziehungswissenschaft. Mitteilungen der deutschen Gesellschaft für Erziehungswissenschaft (DGfE), 26(50), 25-36.

Gieseke, W. (2008). Bedarfsorientierte Angebotsplanung in der Erwachsenenbildung. Bielefeld: Bertelsmann.

Gieseke, W., \& Heuer, U. (2011). Weiterbildungsentscheidungen und beigeordnete Bildung. In R. Arnold (Hrsg.), Lernen im Lebenslauf (S. 107-127). Baltmannsweiler: Schneider Verlag Hohengehren.

Grüner, H. (2000). Die Bestimmung des betrieblichen Weiterbildungsbedarfs. Eine betriebspädagogische Untersuchung am Beispiel gewerblich-mittelständischer Unternehmungen. Frankfurt am Main: Peter Lang.

Harney, K. (1996). Handlungslogik betrieblicher Weiterbildung. Stuttgart: Hirzel.

Herbrechter, D., \& Schrader, J. (2016). Organisationstheoretische Ansätze in der Erwachsenenbildung. In R. Tippelt \& A. v. Hippel (Hrsg.), Handbuch Erwachsenenbildung/Weiterbildung (6. Aufl. S. 1-25). Wiesbaden: Springer.

Heuer, U. (2010). Betriebliche Weiterbildungsentscheidungen: Aushandlungsprozesse und Bildungscontrolling. Fallstudienbericht. In Bundesinstitut für Berufsbildung (Hrsg.): Wissenschaftliche Diskussionspapiere, Heft 115. http://www.bibb.de/veroeffentlichungen/de/publication/download/id/6221. Zugegriffen: 10. Jan 2017.

von Hippel, A. (2016). Programmplanungskulturen in der betrieblichen Weiterbildung. Eine Programmanalyse zu Funktionen betrieblicher Weiterbildungsprogramme. In O. Dörner, C. Iller, H. Pätzold \& S. Robak (Hrsg.), Differente Lernkulturen - regional, national, transnational. Schriftenreihe der Sektion Erwachsenenbildung der DGfE (S. 139-159). Opladen, Berlin, Toronto: Barbara Budrich.

von Hippel, A., \& Röbel, T. (2016). Funktionen als akteursabhängige Zuschreibungen in der Programmplanung betrieblicher Weiterbildung. Zeitschrift für Weiterbildungsforschung, 39(1), 1-21.

Jansen, T., von Schlippe, A., \& Vogd, W. (2015). Kontexturanalyse - ein Vorschlag für rekonstruktive Sozialforschung in organisationalen Zusammenhängen. Forum Qualitative Sozialforschung, 16(1), 4.

Jechle, T., \& Kolb, M. (1994). Bedarfsermittlung in der Weiterbildung. Unterrichtswissenschaft, 22(1), 3-22.

John, R. (2010). Funktionale Analyse - Erinnerungen an eine Methodologie zwischen Fixierung und Überraschung. In R. John, A. Henkel \& J. Rückert-John (Hrsg.), Die Methodologien des Systems. Wie kommt man zum Fall und wie dahinter? (S. 29-54). Wiesbaden: Springer.

Käpplinger, B. (2010). Weiterbildungsentscheidungen auf Grundlage von Bildungscontrolling und jenseits von Bildungscontrolling - Empirische Ergebnisse eines BIBB-Forschungsprojektes. In B. Käpplinger (Hrsg.), Weiterbildungsentscheidungen und Bildungscontrolling. Impulse aus der Bildungsforschung für die Bildungspraxis Wissenschaftliche Diskussionspapiere aus dem BIBB, 117. (S. 6-17). Bonn: BIBB. 
Käpplinger, B. (2013). Ambivalenzen in der betrieblichen Weiterbildung. Hochkonjunktur in der Politik, Stagnation und Expansion in der Empirie, Defizite in der Theorie. In H. v. Felden (Hrsg.), Erwachsenbildung im Spannungsfeld von Wissenschaft, Politik und Praxis (S. 116-128). Baltmannsweiler: Schneider.

Käpplinger, B. (2016a). Theorien und Theoreme der betrieblichen Weiterbildung. In R. Tippelt \& A. von Hippel (Hrsg.), Handbuch Erwachsenenbildung/Weiterbildung 6. Aufl. Wiesbaden: Springer.

Käpplinger, B. (2016b). Betriebliche Weiterbildung aus der Perspektive von Konfigurationstheorien (1. Aufl.). Bielefeld: Bertelsmann.

Lüdecke, D. (2016). Der Einsatz der Dokumentarischen Methode zur Analyse professionell-organisierter Systeme. In M. Hülsken-Giesler, S. Kreutzer \& N. Dütthorn (Hrsg.), Rekonstruktive Fallarbeit in der Pflege. Methodologische Reflexionen und praktische Relevanz für Pflegewissenschaft, Pflegebildung und die direkte Pflege (S. 163-186). Göttingen: Universitätsverlag Osnabrück im Verlag V\&R unipress.

Mayring, P. (2003). Qualitative Inhaltsanalyse. Grundlagen und Techniken. Weinheim: Beltz.

Pohlmann, C. (2015). Angebotsplanung von Bildungsurlaubsveranstaltungen im Spannungsfeld gesellschaftlicher Ansprüche, erwachsenenpädagogischer Ansprüche, erwachsenenpädagogischer Prinzipien und ökonomischer Interessen. In S. Robak, H. Rippien, L. Heidemann \& C. Pohlmann (Hrsg.), Bildungsurlaub - Planung, Programm und Partizipation. Eine Studie in Perspektivverschränkung (S. 69-112). Frankfurt am Main: Peter Lang.

Reich-Claassen, J., \& von Hippel, A. (2009). Angebotsplanung und -gestaltung. In R. Tippelt \& A. von Hippel (Hrsg.), Handbuch Erwachsenenbildung/Weiterbildung (S. 1003-1015). Wiesbaden: Springer VS.

Robak, S. (2012). Programmanalysen: Einführung in die Erstellung von Codesystemen. DIE Bonn. http://www.die-bonn.de/Institut/Dienstleistungen/Servicestellen/Programmforschung/Methodische_ Handreichungen/codiersysteme. Zugegriffen: 10. März 2017.

Robak, S., Pohlmann, C., \& Heidemann, L. (2013). Zur Analyse von Folgewirkungen bildungspolitischer Strukturentscheidungen auf Bildungs- und Planungspartizipation am Beispiel von Bildungsurlaub. In S.M. Weber, M. Göhlich, A. Schröer, C. Fahrenwald \& H. Macha (Hrsg.), Organisation und Partizipation. Beiträge der Kommission Organisationspädagogik (S. 303-313). Wiesbaden: Springer VS.

Röbel, T. (2016). The interaction of economic and pedagogical ideals in the context of workplace learning in Germany: a framework for empirical research - inspired by business ethics. European Journal for Research on the Education and Learning of Adults - RELA, 7(2), 207-221. doi:10.3384/rela.20007426.201672).

Schlutz, E. (2006). Bildungsdienstleistungen und Angebotsentwicklung. Münster: Waxmann.

Schrader, J. (2010). Reproduktionskontexte der Weiterbildung. Zeitschrift für Pädagogik, 56(2), 267-284.

Seusing, B., \& Bötel, C. (2000). Bedarfsanalyse - die betriebliche Praxis der Planung von Weiterbildungsbedarfen. In C. Bötel \& E. Krekel (Hrsg.), Bedarfsanalyse, Nutzenbewertung und Benchmarking zentrale Elemente des Bildungscontrollings (S. 21-34). Bielefeld: Bertelsmann.

Sork, T. J. (1990). An "ethical practices analysis" for adult educators. Vortrag bei der Annual Conference of the American Association for Adult and Continuing Education (AAACE), Salt Lake City.

Staudt, E. (1990). Defizitanalyse betrieblicher Weiterbildung. In W. Schlaffke (Hrsg.), Tendenzen betrieblicher Weiterbildung. Aufgaben für Forschung und Praxis. Köln: Dt. Inst.-Verl.

Vogd, W. (2009). Systemtheorie und Methode? Zum komplexen Verhältnis von Theoriearbeit und Empirie in der Organisationsforschung. Soziale Systeme, 15(1), 97-136.

Vogd, W., Harth, J., \& Ofner, U. (2015). Doing religion im Phowa-Kurs: Praxeologische und reflexionslogische Studien zum ,bewussten Sterben“ im Diamantweg-Buddhismus. Forum: Qualitative Social Research, 16(3), Art. 17.

Weber, W. (1985). Betriebliche Weiterbildung. Empirische Analyse betrieblicher und individueller Entscheidungen über Weiterbildung. Stuttgart: Poeschel.

Weber, W., Mayrhofer, W., Nienhäuser, W., Rodenuth, M., \& Rüther, B. (1994). Betriebliche Bildungsentscheidungen: Entscheidungsverläufe und Entscheidungsergebnisse. München: Rainer Hampp.

Wittwer, W. (1981). Die Legitimation von Zielen in der betrieblichen Weiterbildung. München: LudwigMaximilians-Universität.

Zalenska, L. (2009). Bildungsbedarfsanalyse in Unternehmen (1. Aufl.). Lohmar, Köln: Eul. 